\title{
Liquid Resin Infusion process monitoring with embedded superimposed long period and short period Bragg grating sensor.
}

\author{
S. Triollett ${ }^{1,2,3}$, L. Robert ${ }^{2,3, a}$, E. M arin ${ }^{1}$, and Y . Ouerdane ${ }^{1}$ \\ ${ }^{1}$ L aboratoire Hubert Curien, UM R CNRS 5516, 42000 Saint Etienne, France \\ ${ }^{2}$ Université de Toulouse; INSA, UPS, M ines A Ibi, ISAE; ICA (Institut Clément A der), CROM eP; \\ Campus J arlard, F-81013 A lbi cedex 09, France \\ ${ }^{3}$ E cole des M ines A lbi, Campus J arlard, F-81013 A Ibi, France
}

\begin{abstract}
We propose here the monitoring of the Liquid Resin Infusion (LRI) process for using a superimposed long period (LPG) and short period (FBG) Bragg grating sensor. Monitoring of such a process is usually made measuring simultaneously temperature and strain by the use of an electro-optical device (FBG-Thermocouple). It has been shown that an applied solicitation is measured by a wavelength shift with a different sensitivity for LPG and FBG; thus strain and temperature influences can be determined separately by measuring corresponding wavelength shifts. The reported configuration is based on the use of these two Bragg gratings types written in the same fibre section, which allows us to discriminate the contributions of the temperature and strain. The sensor is embedded in a composite specimen manufactured by LRI process for monitoring in real time and simultaneously the applied temperature and strain.
\end{abstract}

\section{Introduction}

Polymer matrix composites are more and more used as structural materials for many applications because of their weight and high strength. However, some well known issues concern for instance the durability evaluation and damage tolerance design [1,2], the characterisation of residual stresses $[3,4]$ as well as the identification of the global mechanical properties of such composite structures [5], that presents obstacles related to the multiplicity of the involved scales, the complexity of the mechanical answers, the ignorance of the material state and the unavoidable properties variability induced by the manufacturing processes. In this way the monitoring of the process can give some answers $[6,7]$. A mong the composite manufacturing processes, the Liquid Resin Infusion (LRI) process is a novel process for polymer matrix composites whose major interests are to lead to quality controlled parts and reduced assembly steps by infusing components and sub-components at the same time. $L R I$ is based on the moulding of high performance composite parts by infusing liquid resin on dry (carbon or glass) preform instead of prepreg fabrics [8]. However, the final properties of the composite parts remain highly dependent on the process parameters such as time, pressure and temperature of the resin, that need to be measured and controlled during the process.

\footnotetext{
a e-mail : L aurent.R obert@ mines-albi.fr
} 
Optical fibre sensors are generally considered as al ternative solutions to measure and localize the amplitudes of applied solicitations, particularly in applications where the information can not be assessed directly. Because of their low intrusivity, optical fibres can be easily embedded into the polymer matrix to perform in-situ and real-time measurements. Within this framework, all the above cited references report the use of optical fibre sensors. A mong them, B ragg gratings based sensor can be considered as one of the most interesting candidate for the deported optical measurement, which under an adequate analyse of the propagated signal in the fibre core, leads to the determination of the applied solicitation, for instance the temperature or the strain.

A standard fibre Bragg grating (FBG) is obtained by creating a sub-micron period refractive index modulation in the core of an optical fibre that acts to couple the fundamental forward propagating mode to the contra-propagating core mode. This coupling occurs at a specific wavelength $\lambda_{b}$, defined by the Bragg condition for the fibre grating, with the FBG acting as a narrow-band reflection filter. The Bragg wavelength is defined by the period of the FBG and the effective index of the propagating mode, with the result that a change in either one of these parameters, induced for example by a change in temperature or strain, changes the wavelength. A LPG consists of hundreds microns period refractive index modulation that promotes coupling between the propagating core mode and co-propagating cladding modes. The attenuation of the cladding modes results in the transmission spectrum containing a series of bands corresponding to the coupling to a different cladding mode, centred at discrete wavelengths. Similarly to the FBGs, these attenuation bands are sensitive to the period and the length of the LPG and the local environment namely temperature and strain [9].

However, the main problem appears when the two solicitations are simultaneously applied on the grating. A lot of techniques have been reported to overcome this problem. One of them consists in the use of two superimposed FBG [10] or only long period gratings (superimposed or not) [11]. In all of those sorts of designs the small differences in sensitivities are a limitation to discriminate strain-temperature effects.

In this study, we set out an experimental method for measuring strain and temperature applied in the composite part during its fabrication by Liquid Resin Infusion (LRI) process [8]. It consists of insitu sensor instrumentation for extracting thermal and mechanical characteristics of the composite material during both the infusion and the curing, based on a dual period fibre Bragg grating [12]. The originality of our technique is based on two superimposed Bragg gratings (FBG + LPG) [13], allowing a very good spatial resolution of the sensor with regard to the grating length.

In the sequel, the sensor principle is detailed. Then the experimental configuration and calibration procedure are discussed. Last, the results obtained with the embedded-sensor during the curing of a pure epoxy resin and the first data concerning the LRI process monitoring are presented.

\section{Sensor principle}

For a FBG, the shift of $\lambda_{b}$ due to some applied strain and temperature, is given by Equation (1):

$$
\Delta \lambda_{b}=K_{\varepsilon} \Delta \varepsilon+K_{T} \Delta T
$$

where the strain sensitivity is defined by $K_{\varepsilon}=\left(1-P_{e}\right) \lambda_{b}^{0}$ and the temperature sensitivity by $K_{T}=(\xi+\alpha) \lambda_{b}^{0}$ where $\Delta \varepsilon$ and $\Delta \mathrm{T}$ are the variations of strain and temperature applied to the gratings, respectively, $\Delta \lambda_{b}$ is the variation of the resonance wavelength and $\lambda_{b}^{0}$ the initial wavelength at zero strain or reference temperature. $\mathrm{P}_{\mathrm{e}}$ is the Pockel coefficient, $\xi$ and $\alpha$ are respectively the thermooptic coefficient and the coefficient of thermal expansion of the optic fibre.

With only Equation (1) the two unknown can not be extracted. The discrimination method is here made with a sensor containing two superimposed Bragg gratings [12]: a FBG and a LPG. Under strain and temperature solicitations, the two resonance wavelengths shift with different variation, so that the relationship between $\Delta \lambda_{\mathrm{bL}}$ (for the LPG), $\Delta \lambda_{\mathrm{bF}}$ (for the FBG), and the applied temperature and strain can be expressed as: 


$$
\left(\begin{array}{l}
\Delta \lambda_{b L} \\
\Delta \lambda_{b F}
\end{array}\right)=\left(\begin{array}{ll}
K_{T L} & K_{\varepsilon L} \\
K_{T F} & K_{\varepsilon F}
\end{array}\right)\left(\begin{array}{l}
\Delta T \\
\Delta \varepsilon
\end{array}\right)
$$

where $\mathrm{K}_{\varepsilon \mathrm{F}}\left(\mathrm{K}_{\varepsilon \mathrm{L}}\right)$ and $\mathrm{K}_{\mathrm{TF}}\left(\mathrm{K}_{\mathrm{TL}}\right)$ are respectively the strain and the temperature sensitivities of the $\mathrm{FBG}$ (resp., the $L P G$ ), assuming that the cross sensitivities can be neglected. It has been proved that the sensitivities of both the LPG and the FBG are different for the same applied solicitation [14]. The solution of Equation (2) exists if

$$
D=K_{T L} \times K_{\varepsilon F}-K_{\varepsilon L} \times K_{T F} \neq 0
$$

leading to a well-conditioned sensitivity matrix. Once the sensitivities coefficients are estimated by experimental measurements, one can deduced the applied variation of strain and temperature.

The gratings are photo-inscribed in a hydrogen-loaded Corning ${ }^{\text {TM }}$ SM F-28 fibre. The photoinscription of the LPG is made using point per point technique. A CW UV laser beam, operating at $244 \mathrm{~nm}$, is focused on the fibre core while the fibre is moved depending on the chosen grating period. Then, the FBG is written using a phase mask with no disassembly of the fibre, over the entire length of the LPG. The interest of this method aims at a better accuracy of the superposition of the two gratings, and the selection of the LPG coupled mode to have the best sensitivity. The stabilization of the final component response is obtained by thermal annealing at a temperature of $160^{\circ} \mathrm{C}$ during 6 hours.

\section{Experimental configuration and calibration}

The interrogation of the sensor is made by a white light OEM supercontinuum source of bandwidth $>700 \mathrm{~nm}$ from Leukos as a probe, and an optical spectrum analyzer (Advantest Q8384) for the detection. This set up provides a better accuracy in monitoring the spectrum due to the power and the broadband of the supercontinuum source. It also presents a great advantage in comparison to ELED sources, which has a small spectral bandwidth or to classical halogen sources which have less power. Signal processing of the transmission spectra is made by specific routines developed under Labview software: a fitting is done by minimization in the least squares sense of the difference between the experimental spectrum for the considered range and a Gaussian function, results to an optimized set of parameters related to the B ragg wavelengths of both gratings. It gives an estimation of the $\lambda_{b}$ shift with a resolution better than $15 \mathrm{pm}$ for the $L P G$ and $1.5 \mathrm{pm}$ for the FBG. In order to calibrate the sensor, a bench (Figure 1) have been designed to impose prescribed axial strain and/or temperature to the optical fibre sensor. The sensitivity matrix elements of Equation (2) are determined by measuring the wavelength shifts induced by strain and temperature separately.

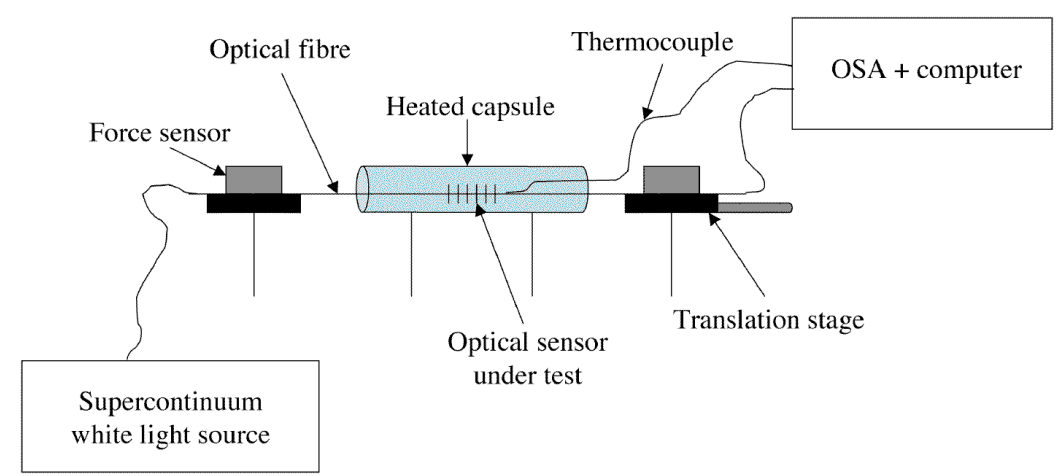

Fig. 1. Schematic diagram of the calibration bench 
The temperature calibration is obtained with a heated capsule and a micro-thermocouple with no strain variation. Concerning the strain, a micrometric translation stage and a micro-force sensor are used at fixed temperature. Figure 2 shows the evolution of the wavelengths shift $\Delta \lambda_{b L}$ and $\Delta \lambda_{b F}$ with the applied temperature in the range $25^{\circ} \mathrm{C}-120^{\circ} \mathrm{C}$ with no applied strain. As expected in this range, it is shown that $\Delta \lambda_{\mathrm{bL}}$ and $\Delta \lambda_{\mathrm{bF}}$ vary linearly with the temperature. The thermal sensitivities $\mathrm{K}_{\mathrm{TL}}=38.9$ $\mathrm{pm} /{ }^{\circ} \mathrm{C}$ and $\mathrm{K}_{\mathrm{TF}}=13.1 \mathrm{pm} /{ }^{\circ} \mathrm{C}$ of both $\mathrm{LPG}$ and $\mathrm{FBG}$ are deduced from the slopes with a linear regression fit (see Figure 2 ).

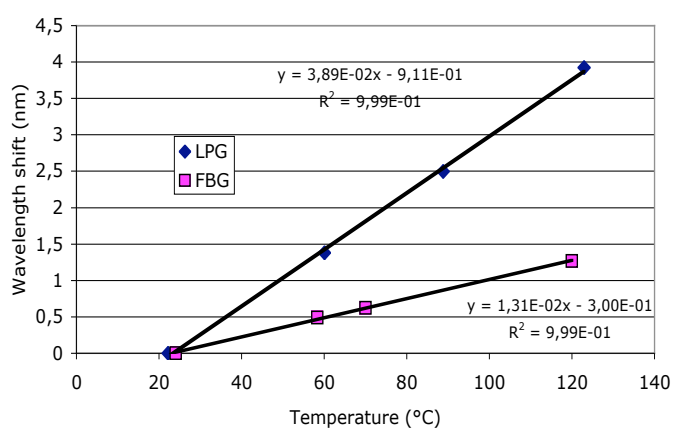

Fig. 2. $L P G$ and $F B G$ wavelengths shift in air as function of temperature with no strain.

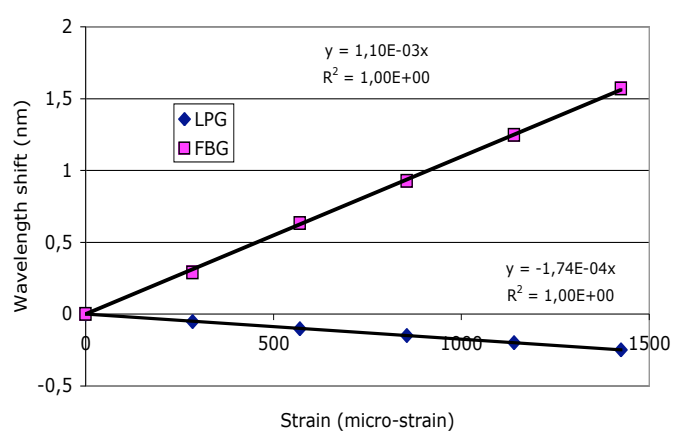

Fig. 3. $L P G$ and $F B G$ wavelengths shift in air as function of strain at $24^{\circ} \mathrm{C}$.

Strain is also applied to the dual period grating in a range $0-1500 \quad \varepsilon$ at room temperature (see Figure 3). The deduced strain sensitivities are $\mathrm{K}_{\varepsilon \mathrm{L}}=-0.17 \mathrm{pm} / \varepsilon$ and $\mathrm{K}_{\varepsilon \mathrm{F}}=1.10 \mathrm{pm} / \varepsilon$ respectively. Using this configuration several strain measurements at several fixed temperatures have been performed [14]. The recorded wavelengths shifts exhibit quasi-parallel curves which reflects a negligible cross sensitivities of both considered solicitations. The errors on the sensitivities are expressed with Equation (4) [16]:

$$
\delta K_{T j}=\sqrt{\frac{1}{N-2}\left[\sum\left(\Delta \lambda_{b j_{i}}-\overline{\Delta \lambda_{b j}}\right)^{2}-\frac{\left[\sum\left(T_{i}-\bar{T}\right)\left(\Delta \lambda_{b j_{i}}-\overline{\Delta \lambda_{b j}}\right)\right]^{2}}{\sum\left(T_{i}-\bar{T}\right)^{2}}\right]}
$$

with $N$ the total number of $i$ samples. $J=L, F$ stands for $L P G$ and $F B G$ respectively and the overline variables represent the mean value. The estimated thermal sensitivities errors lead to $0.8 \mathrm{pm} /{ }^{\circ} \mathrm{C}$ and $0.3 \mathrm{pm} /{ }^{\circ} \mathrm{C}$ for $\mathrm{K}_{\mathrm{TL}}$ and $\mathrm{K}_{\mathrm{TF}}$ respectively. Using Equation (4) while replacing temperature by strain, we found strain sensitivities errors to be $0.03 \mathrm{pm} / \varepsilon$ and $0.01 \mathrm{pm} / \varepsilon$ for $\mathrm{K}_{\varepsilon \mathrm{L}}$ and $\mathrm{K}_{\varepsilon \mathrm{F}}$ respectively.

\section{Results and discussions}

\subsection{Simple epoxy resin curing monitoring}

In a first application, the sensor has been embedded in an Hexcel RTM 6 epoxy resin for curing monitoring purpose. A micro-thermocouple of $110 \mathrm{~m}$ wire diameter has been positioned in the vicinity of the optical sensor in order to validate and compare the results. Some studies reported the use of a single FBG and a thermocouple to discriminate temperature from strain during composite process and to achieve determination of residual strains (see e.g. [4]). However, the device presented here seems to be a good way to be free from the use of an invasive (metal-based) thermocouple. In situ acquisition has been performed during all the curing process. Figure 4 presents the temperature 
measured by the thermocouple embedded near the optical sensor for comparison and the FBG and LPG wavelength shifts function of time during the process.

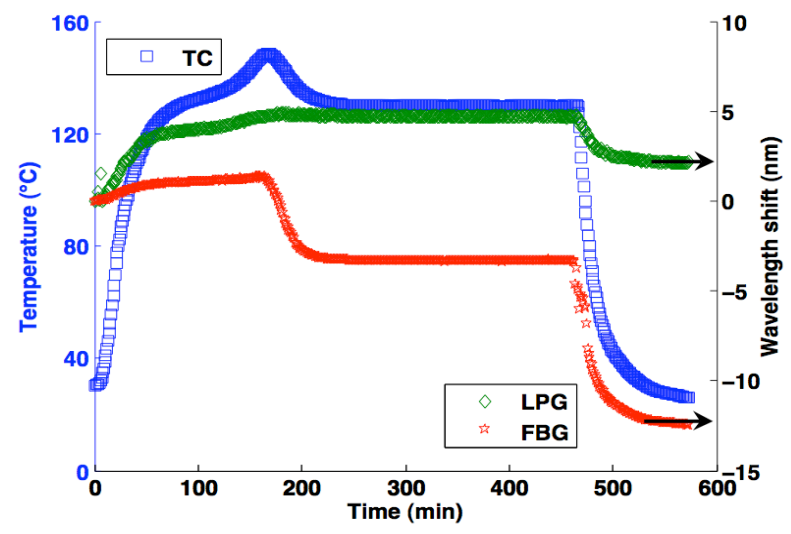

Fig. 4. Thermocouple temperature (left), FBG and LPG wavelength shifts (right) during epoxy resin curing

The process shown in Figure 4 may be cut in 3 steps: the temperature increase, the reticulation phase (cure plateau), and the temperature decrease. In the first step, during the temperature increase from $30^{\circ} \mathrm{C}$ to $130^{\circ} \mathrm{C}$ (at around $2^{\circ} \mathrm{C} / \mathrm{min}$ ), the epoxy resin can be considered as liquid, so there is no sensible mechanical strain applied on the dual grating $(\varepsilon=0)$. The measured shift of $\lambda_{b}$ only depends on temperature, which can be used as an in situ temperature calibration including its environment; this means that the shift of $\lambda_{b}$ may be written as Equation (5) with $K_{T}$ known from calibration:

$$
\Delta \lambda_{b}=K_{T} \Delta T
$$

During the reticulation phase (second step), we may observed two distinct phases. The first one is an overshoot of the temperature which corresponds to the exothermal manifestation of the chemical bondings, while the second phase can be considered as isothermal at $130^{\circ} \mathrm{C}$. However, in general cases, the overshoot does not appear because the reticulation of the resin is made at constant temperature. In our experiment, this leads to unknown values $\Delta T \neq 0$ and $\varepsilon \neq 0$, because Equation ( 1 ) can not be solved when considering that the temperature sensitivity remains unknown during this overshoot. In fact, the epoxy resin continuously changes from its liquid phase to its reticulated phase (which in the same time is going to fix to the optical fibre), leading to a continuously change of the behaviour of the surrounded material. The total strain applied here is the sum of the mechanical part and the thermal one, which is observed by the contraction of the material: negative and positive $B$ ragg wavelength shift for respectively the $F B G$ and $L P G$ in Figure 4.

During the isothermal phase $(\Delta T=0)$ the shift is expressed by Equation (6), which is only dependent of the mechanical strain:

$$
\Delta \lambda_{b}=K_{\varepsilon} \Delta \varepsilon
$$

The process ends after the cure plateau, with the decrease of temperature (third step), leading to residual cure stresses proportional to the measured residual strains [4, 6]. In this case, the Bragg wavel ength depends on strain and temperature, as

$$
\Delta \lambda_{b}=K_{\varepsilon} \Delta \varepsilon+K_{T} \Delta T+K_{\varepsilon}\left(\alpha_{\text {media }}-\alpha_{S i O_{2}}\right) \Delta T=K_{\varepsilon} \Delta \varepsilon+K_{T}^{*} \Delta T
$$

The additional thermal strain effect induces generally an increase of the temperature sensitivity, leading to the apparent temperature sensitivity $K_{T}^{*}=K_{T}+K_{\varepsilon}\left(\alpha_{\text {media }}-\alpha_{S_{i O_{2}}}\right)$ linked to the surrounding 
media. From the curves of the FBG and LPG wavelength shifts presented in Figure 4 discrimination can then be done knowing the temperature and strain sensitivities from calibration. Figure 5 shows the temperature response of our sensor (quoted LPG\&FBG in Figure 5) during the curing process while the total strain is reported in Figure 6.

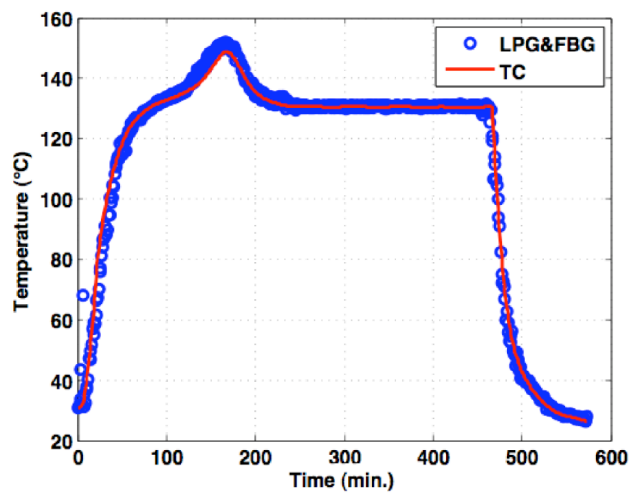

Fig. 5. Temperature measured with the superimposed LPG/FBG sensor during the curing process

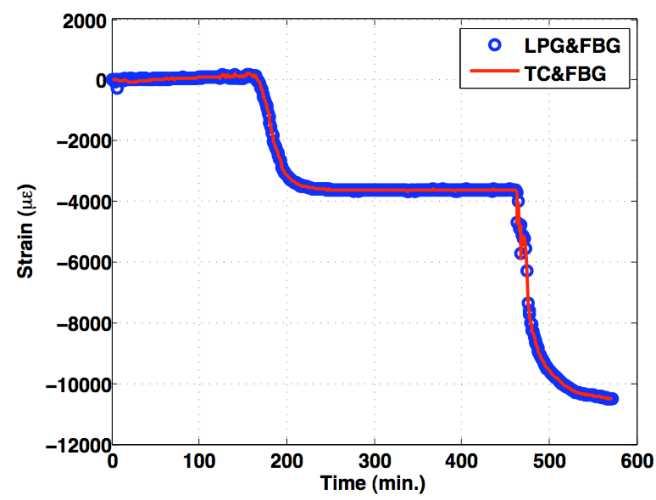

Fig. 6. Strain measured with the superimposed LPG/FBG sensor during the curing process

The results presented in Figure 5 are validated by comparison with the thermocouple response for temperature (quoted TC in Figure 5). The results for the strain measurement presented in Figure 6 are also validated by comparing the strain measured with our optical sensor and the strain deduced from the single FBG wavelength shift using the thermocouple to measure the temperature and to discriminate both solicitations. It is worth pointing out that these results are quite very good except for the temperature response during the overshoot, where the optical sensor and thermocouple responses differ briefly. This may be explained by an underestimate of the thermocouple response, which has some inertia due to current transit and produces local temperature flux.

\subsection{Liquid Resin Infusion (LRI) monitoring}

The superimposed Bragg gratings sensor has been embedded in a glass/epoxy composite part made using the $L R I$ process.

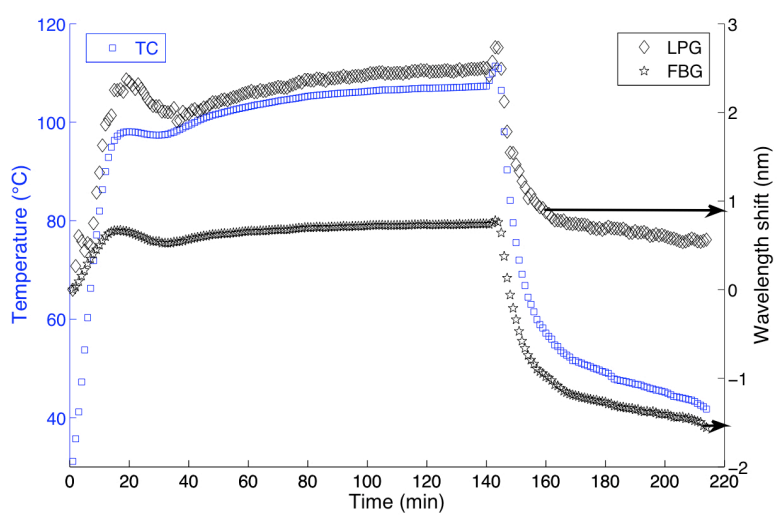

Fig. 7. Thermocouple temperature (left), FBG and LPG wavel ength shifts (right) during the LRI process 
Figure 7 reports the wavelength shifts of both gratings (LPG and FBG) that have been acquired during all the process and the temperature measured by a thermocouple for comparison. The process can be cut also in 3 steps: the temperature increase, the cure plateau and the temperature decrease. A s it can be seen in Figure 7, during the cure plateau the imposed temperature was not being well controlled and a small continuous temperature increase is observed from 35 minutes up to the end of the cure plateau. However, as previously shown in Section 4.1 for the resin cure, temperature and strain may be discriminated knowing the sensitivities of both gratings from calibration.

Figure 8 reports the results of the measured LPG\&FBG sensor temperature function of time, while strain is presented in Figure 9.

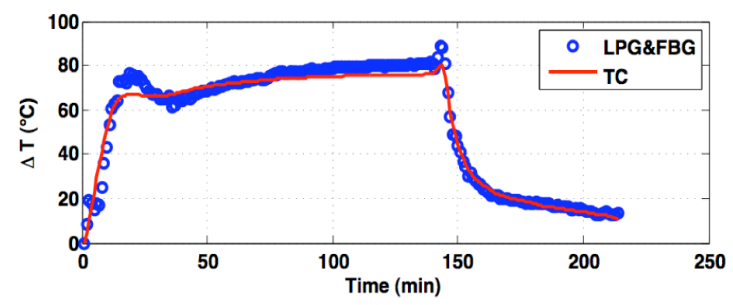

Fig. 8. Temperature measured with the superimposed $L P G / F B G$ sensor during the $L R I$ process

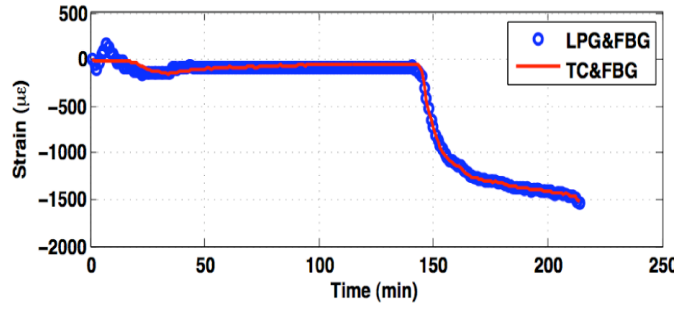

Fig. 9. Strain measured with the superimposed LPG/FBG sensor during the LRI process

It is worth noting that measured temperature values exhibit differences and do not fit well mainly during the reticulation phase. Actually, considering these first results, we can not explain exactly the differences in behaviour between the superimposed optical sensor and thermocouple responses. We can not neglect the fact that both sensors are not really positioned at the same spatial location inside the perform, and that thermocouple is not a standard temperature device. However, one way to discuss these results is to plot the wavelengths shifts of both gratings as function of the thermocouple temperature, as presented in Figure 10 and 11 upon which are reported the 3 phases of the $L R I$ process.

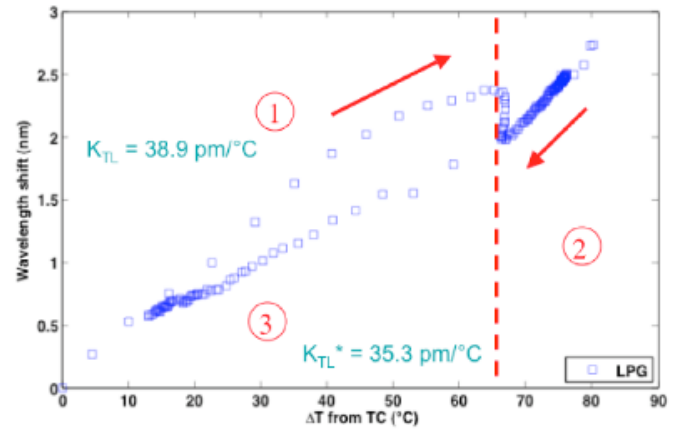

Fig. 10. LPG wavelength shift as function of temperature measured by thermocouple

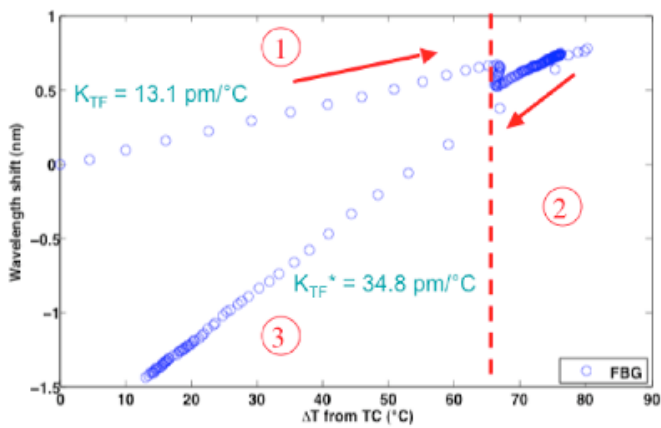

Fig. 11. FBG wavelength shift as function of temperature measured by thermocouple

During the increase of temperature (step 1), the wavelengths are continuously increasing with the sensitivities $K_{T}$ obtained from calibration in air. The reticulation phase (step 2) presents a decrease of wavelengths during the isothermal part, followed by an increase of both the temperature and the wavelengths because of the non-controlled process imposed temperature. The process ends 
with the decrease of temperature (step 3) with a final slope $K_{T}^{*}$ which takes into account the third term of Equation (7) because the composite is totally cured. The coefficient of thermal expansion of the composite material thus can be evaluated using Equation (7) and is found to be $\alpha_{\text {media }} \approx 20 \mu \varepsilon /{ }^{\circ} \mathrm{C}$. The non well-defined behaviour appears during step 2 (see Figure 10): the first (isothermal) phase of step 2, as discussed previously, should presents a contraction of the material, which is in contradiction with the decrease in wavelength observed in Figure 10 while keeping negative strain sensitivity of the LPG.

\section{Conclusion}

We reported an optical fibre sensor based on dual period B ragg gratings, which can be used for discrimination of simultaneous strain and temperature measurements at the same spatial position. Our sensor has been calibrated to get sensitivities response before embedment in epoxy resin, then in glass/epoxy composite specimen for LRI process monitoring. The discrimination of temperature and strain response of the sensor has been validated during a simple epoxy resin curing by comparison with thermocouple measurement. It presents a good accurate response for the monitoring and a small intrusivity while give up the use of thermocouple. LRI process has also been monitoring and responses of our sensor exhibit differences from the ones acquired with thermocouple, which are still under study. This component type can then be implemented in an in-situ fabrication control process for monitoring the solicitations applied to the composite materials.

\section{References}

1. N. Takeda, S. Ogihara, Comp. Sci. Tech. 52, 309 (1994)

2. N. Takeda, Int. J. Fatigue 24, 281 (2002)

3. F. Colpo, L. Humbert, J. B otsis, Comp. Sci. Tech. 67, 1830 (2007)

4. M. M ulle, F. Collombet, P. Olivier, Y.-H. Grunevald, Comp. Part A 40, 1534 (2009)

5. M. Mulle, R. Zitoune, F. Collombet, L. Robert, Y .-H. Grunevald, Comp. Struct. 91, 48 (2009)

6. M.J. O'D wyer, G.M. M aistros, S.W. James, R.P. Tatam, I.K. Partridgez, M eas. Sci. Tech. 9, $1153(1998)$

7. Y. Wang, B. Han, D.W. Kim, A. Bar-Cohen, P. J oseph, Exp. M ech. 48, 107 (2008)

8. C.W illiams, J. Summerscales, S. Grove, Comp. Part A 27, 517 (1996)

9. V. B hatia, D. Campbell, R.O. Claus, Opt. Lett. 22, 648 (1997)

10. M.G. X u, J.L. A rchambault, L. Reekie, J.P. Dakin, Electron. L ett. 30, 1085 (1994)

11. H.G. Han, S.B. Lee, C.S. Kim, J.U. Kang, U.C. Paek, Y. Chung, Opt. Express 11, 476 (2003)

12. M. Wang, T. Li, Y. Zhao, H. W ei, Z. Thong, S. Jian, Proc. SPIE 4579 (2001)

13. S. Triollet, L. Robert, E. M arin, Y. Ouerdane, submitted to Sens. Act. A (2010)

14. V. Bhatia, Opt. Express 4, 457 (1999)

15. S. Triollet, L. R obert, E. M arin, Y. Ouerdane, in Proc. OF S20, Edinburgh, UK (2009).

16. M. N euilly, Tech. Ing. R-280 (1987) 